\title{
Integration of Lie 2-Algebras and Their Morphisms
}

\author{
YUNHE SHENG ${ }^{1}$ and CHENCHANG ZHU ${ }^{2}$ \\ ${ }^{1}$ Department of Mathematics, Jilin University, Changchun 130012, Jilin, China. \\ e-mail: shengyh@jlu.edu.cn \\ ${ }^{2}$ Mathematischen Institut and Courant Research Centre "Higher Order Structures", \\ University of Göttingen, Göttingen, Germany. e-mail: zhu@uni-math.gwdg.de
}

Received: 24 September 2011 / Revised: 4 May 2012 / Accepted: 27 June 2012

Published online: 28 July 2012 - (C) The Author(s) 2012. This article is published with open access at Springerlink.com

\begin{abstract}
Given a strict Lie 2-algebra, we can integrate it to a strict Lie 2-group by integrating the corresponding Lie algebra crossed module. On the other hand, the integration procedure of Getzler and Henriques will also produce a 2-group. In this paper, we show that these two integration results are Morita equivalent. As an application, we integrate a non-strict morphism between Lie algebra crossed modules to a generalized morphism between their corresponding Lie group crossed modules.
\end{abstract}

Mathematics Subject Classification (2010). Primary 17B55; Secondary 18B40, 18D10.

Keywords. $L_{\infty}$-algebras, $L_{\infty}$-morphisms, crossed modules, Lie 2-groups, Lie 2-algebras, integration.

\section{Introduction}

Recently people have paid much attention to the integration of Lie-algebra-like structures, such as that of Lie algebroids [7,9,27], of $L_{\infty}$-algebras [13,14] and of Courant algebroids [17,19,24]. Here "integration" is meant in the same sense in which a Lie algebra is integrated to a corresponding Lie group.

For an $L_{\infty}$-algebra $\mathfrak{h}$, there is an infinite dimensional Kan simplicial space $\int \mathfrak{h}$ constructed in $[13,14]$, whose $k$-cells are given by $L_{\infty}$-algebroid morphisms $T \Delta^{k} \rightarrow$ $\mathfrak{h}$. Applying to a strict Lie 2-algebra $\mathfrak{h}$, the 2-truncation $\tau_{2}\left(\int \mathfrak{h}\right)$ is a 2-group which is believed to be the universal integration of $\mathfrak{h}$.

On the other hand, a strict Lie 2-algebra (resp. strict Lie 2-group) one-to-one corresponds to a Lie algebra (resp. Lie group) crossed module. Thus, a strict Lie 2-algebra can be easily integrated to a strict Lie 2-group by integrating its corresponding crossed module. In this article, we prove that these two integration results are Morita equivalent. Already noticed in [4], the classifying Postnikov data of Lie

\footnotetext{
Y. Sheng is supported by NSFC (11101179) and SRFDP (20100061120096). C. Zhu is supported by the German Research Foundation (Deutsche Forschungsgemeinschaft (DFG)) through the Institutional Strategy of the University of Göttingen.
} 
2-algebras is the quotient Lie algebra in degree 0 , a choice of Lie module and of a corresponding 3-cocycle. When the Lie module structure is trivial, as in the case of string Lie 2-algebra, the above Morita equivalence may be implied via a homotopy theoretical method (see [11, Section 4.1.3]). Our article further provides an explicit Morita morphism generally regardless the triviality of the Lie module. We must warn the readers that we treat finite dimensional case only because we need to use the fact that the second homotopy group of a Lie group is trivial, and this is true only in the finite dimensional case.

As $L_{\infty}$-algebras and their integration play an important role in higher gauge theory [5] and higher Chern-Weil theory [11], we believe our explicit construction will have potential application in mathematical physics.

As an application, we use the above result to integrate a nonstrict morphism between strict Lie 2-algebras to a generalized morphism between their strict Lie 2-groups. We must mention that an integration of such morphisms is also provided via the technique of butterflies in [20]. Here we also provide some mathematical physics oriented examples of such morphisms: they can encode 2-term $L_{\infty}$-modules of $\mathfrak{g}$ in the sense of [16], or equivalently, 2-term representation up to homotopy in the sense of [2], non-abelian extensions of $\mathfrak{g}$, and up to homotopy Poisson actions of $\mathfrak{g}$ in the sense of [21]. Further application of the integration is provided in [23].

\section{Equivalence of Integrations}

For an $L_{\infty}$-algebra $\mathfrak{h}$, there is an infinite dimensional Kan simplicial space $\int \mathfrak{h}$ constructed in $[13,14]$,

$$
\mathfrak{h}_{k}:=\operatorname{Hom}_{\text {d.g.c.a. }}\left(\wedge^{\bullet}(\mathfrak{h}), \Omega^{\bullet}\left(\Delta_{k}\right)\right) .
$$

Here we remind the readers that $\wedge^{\bullet}(\mathfrak{h})$ has a natural differential graded commutative algebra (d.g.c.a.) structure which generalizes the Chevalley-Eilenberg complex for a Lie algebra. It is shown in this paper, that when $\mathfrak{h}$ is a Lie algebra, the one-truncation $\tau_{1}(\mathfrak{h})$ is exactly the nerve of the simply connected Lie group $H$ integrating $\mathfrak{h}$.

When $\mathfrak{h}$ is a strict Lie 2-algebra corresponding to the crossed module $\mathfrak{h}_{1} \stackrel{\mathrm{d}}{\rightarrow} \mathfrak{h}_{0}$, the two-truncation $\tau_{2}(\mathfrak{h})$ is a 2-group. On the other hand, there is another natural Lie 2-group corresponding to the integrated crossed module of Lie groups (see Definition 2.6) $H_{1} \stackrel{\text { d }}{\rightarrow} H_{0}$, where $H_{0}$ and $H_{1}$ are simply connected Lie groups of $\mathfrak{h}_{0}$ and $\mathfrak{h}_{1}$ respectively (for this integration see for example [23, Remark 3.7]). In this section, we show the isomorphisms between these two 2-groups.

\subsection{BACKGROUND ON $L_{\infty}$ ALGEBRAS}

In this section, we briefly review the notions of $L_{\infty}$-algebras and crossed modules of Lie algebras. They both provide models for strict Lie 2-algebras. 
$L_{\infty}$-algebras, sometimes called strongly homotopy Lie algebras, were introduced by Stasheff [25] as a model for "Lie algebras that satisfy Jacobi identity up to all higher homotopies". The following convention of $L_{\infty}$-algebras is the same as Lada and Mark1 [16].

DEFINITION 2.1. An $L_{\infty}$-algebra is a graded vector space $L=L_{0} \oplus L_{1} \oplus \cdots$ equipped with a system $\left\{l_{k} \mid 1 \leq k<\infty\right\}$ of linear maps $l_{k}: \wedge^{k} L \longrightarrow L$ with degree $\operatorname{deg}\left(l_{k}\right)=k-2$, where the exterior powers are interpreted in the graded sense and the following relation with Ksozul sign "Ksgn" is satisfied for all $n \geq 0$ :

$$
\begin{aligned}
& \sum_{i+j=n+1}(-1)^{i(j-1)} \sum_{\sigma} \operatorname{sgn}(\sigma) \operatorname{Ksgn}(\sigma) l_{j}\left(l_{i}\left(x_{\sigma(1)}, \ldots, x_{\sigma(i)}\right),\right. \\
& \left.x_{\sigma(i+1)}, \ldots, x_{\sigma(n)}\right)=0
\end{aligned}
$$

where the summation is taken over all $(i, n-i)$-unshuffles with $i \geq 1$.

If $L$ is concentrated in degrees $<n$, we obtain the notion of $n$-term $L_{\infty}$-algebras. A semi-strict Lie 2-algebra can be understood as a 2-term $L_{\infty}$-algebra. a strict Lie 2-algebra is a 2-term $L_{\infty}$-algebra, in which $l_{3}$ is zero (see [4]).

DEFINITION 2.2. A crossed module of Lie algebras is a quadruple $\left(\mathfrak{h}_{1}, \mathfrak{h}_{0}, \mathrm{~d} t, \phi\right)$, where $\mathfrak{h}_{1}$ and $\mathfrak{h}_{0}$ are Lie algebras, $\mathrm{d} t: \mathfrak{h}_{1} \longrightarrow \mathfrak{h}_{0}$ is a Lie algebra morphism and $\phi$ : $\mathfrak{h}_{0} \longrightarrow \operatorname{Der}\left(\mathfrak{h}_{1}\right)$ is an action of Lie algebra $\mathfrak{h}_{0}$ on Lie algebra $\mathfrak{h}_{1}$ as a derivation, such that

$$
\mathrm{d} t\left(\phi_{u}(m)\right)=[u, \mathrm{~d} t(m)]_{\mathfrak{h}_{0}}, \quad \phi_{\mathrm{d} t(m)}(n)=[m, n]_{\mathfrak{h}_{1}} .
$$

Here $\operatorname{Der}\left(\mathfrak{h}_{1}\right)$ is the derivation Lie algebra of $\mathfrak{h}_{1}$ with the commutation Lie bracket $[\cdot, \cdot]_{C}$.

The following result is well known.

THEOREM 2.3. There is a one-to-one correspondence between strict Lie 2-algebras and crossed modules of Lie algebras.

For the precise relation between the operation $l_{2}$ and the Lie brackets $[\cdot, \cdot]_{\mathfrak{h}_{0}}$ and $[\cdot, \cdot]_{\mathfrak{h}_{1}}$, please see [23]. The key difference is that $l_{2}(m, n)=0$, for any $m, n \in L_{1}=\mathfrak{h}_{1}$, and $[m, n]_{\mathfrak{h}_{1}}=l_{2}(\mathrm{~d} m, n) \neq 0$. On the direct sum $\mathfrak{h}_{0} \oplus \mathfrak{h}_{1}$, there is also a Lie bracket $[\cdot, \cdot]_{\mathfrak{h}_{0} \oplus \mathfrak{h}_{1}}$, which is the semidirect product of the Lie algebra $\mathfrak{h}_{0}$ and the Lie algebra $\mathfrak{h}_{1}$ :

$$
[u+m, v+n]_{\mathfrak{h}_{0} \oplus \mathfrak{h}_{1}}=l_{2}(u, v)+l_{2}(u, n)+l_{2}(m, v)+[m, n]_{\mathfrak{h}_{1}} .
$$


EXAMPLE 2.4. For any Lie algebra $\mathfrak{k},(\mathfrak{k}, \operatorname{Der}(\mathfrak{k})$, ad, Id) is a crossed module of Lie algebras. We denote by $\mathfrak{k} \stackrel{\text { ad }}{\longrightarrow} \operatorname{Der}(\mathfrak{k})$ the corresponding strict Lie 2-algebra.

\subsection{BACKGROUND ON 2-GROUPS}

A group is a monoid where every element has an inverse. A 2-group is a monoidal category where every object has a weak inverse and every morphism has an inverse. Denote the category of smooth Banach manifolds and smooth maps by Diff, a semistrict Lie 2-group is a 2-group in DiffCat, where DiffCat is the 2-category consisting of categories, functors, and natural transformations in Diff. In the sequel, all the Lie 2-groups are semistrict.

DEFINITION 2.5. A semistrict Lie 2-group consists of an object $C$ in DiffCat together with

- a multiplication morphism (horizontal multiplication) ${ }^{\cdot h}: C \times C \longrightarrow C$,

- identity object 1 ,

- an inverse map inv $: C \longrightarrow C$

together with the following natural isomorphisms::

- the associator

$$
a_{x, y, z}:(x \cdot \mathrm{h} y) \cdot \mathrm{h} z \longrightarrow x \cdot \mathrm{h}(y \cdot \mathrm{h} z),
$$

- the left and right unit

$$
l_{x}: 1 \cdot \mathrm{h} x \longrightarrow x, \quad r_{x}: x \cdot \mathrm{h} 1 \longrightarrow x,
$$

- the unit and counit

$$
i_{x}: 1 \longrightarrow x \cdot h \operatorname{inv}(x), \quad e_{x}: \operatorname{inv}(x) \cdot h x \rightarrow 1,
$$

such that the pentagon identity for the associator, the triangle identity for the left and right unit, the first and second zig-zag identities are satisfied. We refer to [3, Definition 7.1].

As pointed out in [3, Section 7], if the category $C$ carries a semistrict Lie 2 -group structure, then $C$ must be a Lie groupoid. We denote the groupoid multiplication in $C$ by $\cdot_{\mathrm{v}}$ (vertical multiplication).

In the special case when $a_{x, y, z}, l_{x}, r_{x}, i_{x}, e_{x}$ are all identity isomorphisms, we obtain the concept of a strict Lie 2-group. It is well-known that strict Lie 2-groups can be described by crossed modules of Lie groups.

DEFINITION 2.6. A crossed module of Lie groups is a quadruple $\left(H_{1}, H_{0}, t, \Phi\right)$, where $H_{0}$ and $H_{1}$ are Lie groups, $t: H_{1} \longrightarrow H_{0}$ is a Lie group morphism, and $\Phi$ : 
$H_{0} \times H_{1} \longrightarrow H_{1}$ is an action of $H_{0}$ on $H_{1}$ as automorphisms of $H_{1}$ such that $t$ is $H_{0}$-equivariant:

$$
t \Phi_{g}(h)=g t(h) g^{-1}, \quad \forall g \in H_{0}, h \in H_{1},
$$

and $t$ satisfies the so called Peiffer identity:

$$
\Phi_{t(h)}\left(h^{\prime}\right)=h h^{\prime} h^{-1}, \quad \forall h, h^{\prime} \in H_{1} .
$$

The following result is well-known, see $[3,12]$ for more details.

THEOREM 2.7. There is a one-to-one correspondence between crossed modules of Lie groups and strict Lie 2-groups.

Roughly speaking, given a crossed module $\left(H_{1}, H_{0}, t, \Phi\right)$ of Lie groups, the corresponding strict Lie 2-group has $C_{0}=H_{0}$ and $C_{1}=H_{0} \ltimes H_{1}$, the semidirect product of $H_{0}$ and $H_{1}$. In this strict Lie 2-group, the source and target maps $s, t: C_{1} \longrightarrow C_{0}$ are given by:

$$
s(g, h)=g, \quad t(g, h)=t(h) \cdot g,
$$

the vertical multiplication $\cdot_{\mathrm{v}}$ is given by:

$$
\left(g^{\prime}, h^{\prime}\right) \cdot{ }_{\mathrm{v}}(g, h)=\left(g, h^{\prime} \cdot h\right), \quad \text { where } \quad g^{\prime}=t(h) \cdot g,
$$

the horizontal multiplication ${ }^{h}$ is given by:

$$
(g, h) \cdot{ }_{\mathrm{h}}\left(g^{\prime}, h^{\prime}\right)=\left(g \cdot g^{\prime}, h \cdot \Phi_{g}\left(h^{\prime}\right)\right) .
$$

DEFINITION 2.8. Given two Lie 2-groups $C$ and $C^{\prime}$, a unital morphism $F: C \longrightarrow$ $C^{\prime}$ consists of a smooth functor $\left(F_{0}, F_{1}\right): C \longrightarrow C^{\prime}$ equipped with a 2-isomorphism

$$
F_{2}(x, y): F_{0}(x) \cdot \mathrm{h} F_{0}(y) \longrightarrow F_{0}(x \cdot \mathrm{h} y),
$$

such that $F_{0}\left(1_{C}\right)=1_{C^{\prime}}$ and the following diagrams commute:

- the compatibility condition of $F_{2}$ with the associator:

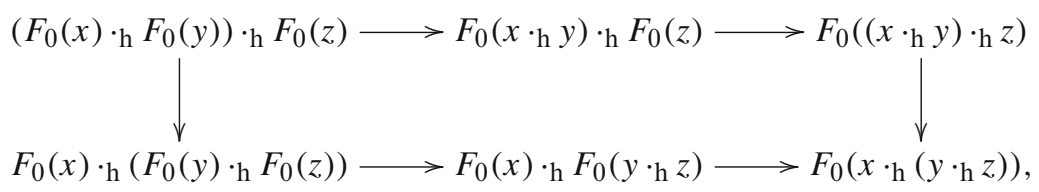


- the compatibility condition of $F_{2}$ with the left and right unit:

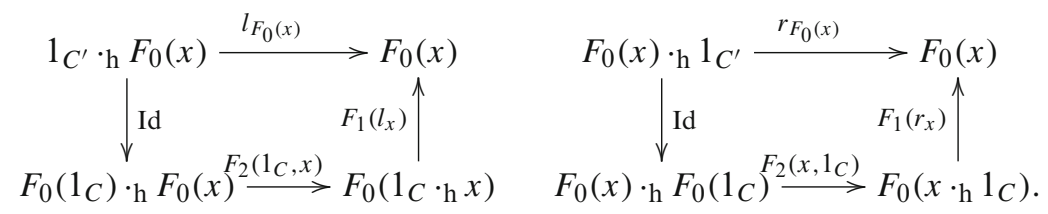

\subsection{EQUIVALENCE OF TWO 2-GROUPS}

Given a Lie algebra $\mathfrak{g}$, denote by $P \mathfrak{g}$ the usual $C^{2}$ path space in $\mathfrak{g}$ and by $P^{0} \mathfrak{g}$ the $C^{2}$ path space in $\mathfrak{g}$ with a convenient boundary condition,

$$
P^{0} \mathfrak{g}=\left\{a: C^{2} \text { morphism from }[0,1] \text { to } \mathfrak{g} \mid a(0)=a(1)=0, a^{\prime}(0)=a^{\prime}(1)=0\right\} .
$$

Then both $P \mathfrak{g}$ and $P^{0} \mathfrak{g}$ naturally have a smooth structure of Banach manifold because we choose $C^{2}$ paths (see for example [27, Section 2]). From now on, when not specially mentioned, all morphisms are of $C^{2}$-classes.

The paths $a_{0}$ and $a_{1}$ are said to be $\mathfrak{g}$-homotopic and we write $a_{0} \sim a_{1}$, if there exist $C^{2}$-morphisms $a, b:[0,1]^{\times 2} \rightarrow \mathfrak{g}$ satisfy the following differential equation

$$
\partial_{t} b(t, s)-\partial_{s} a(t, s)=[a(t, s), b(t, s)]_{\mathfrak{g}}
$$

with boundary value $b(0, s)=0, b(1, s)=0, a(t, 0)=a_{0}(t)$ and $a(t, 1)=a_{1}(t)$. This is equivalent [8] to the fact that,

$$
a(t, s) \mathrm{d} t+b(t, s) \mathrm{d} s: T I \times T I \longrightarrow \mathfrak{g}
$$

is a Lie algebroid morphism and $b(0, s)=b(1, s)=0$. The $\mathfrak{g}$-homotopy also restricts to $P^{0} \mathfrak{g}$ (see [27]). Then the simply connected Lie group $G$ of $\mathfrak{g}$ is the quotient

$$
G \cong P^{0} \mathfrak{g} / \sim=P \mathfrak{g} / \sim:=\tau_{1}\left(\int \mathfrak{g}\right),
$$

(see [10, Section 1.13] for more details).

Next, we recall the construction ([14]) of the 2-group structure of $\tau_{2}\left(\int \mathfrak{h}\right)$ for a strict Lie 2-algebra $\mathfrak{h}$. Let

$$
P_{1} \mathfrak{h}:=\left\{a: C^{2} \text { morphism }[0,1] \rightarrow \mathfrak{h}\right\}=\left\{a: C^{2} \text { morphism }[0,1] \rightarrow \mathfrak{h}_{0}\right\},
$$

and

$$
\begin{array}{r}
P_{2} \mathfrak{h}:=\left\{(a, b, z):[0,1]^{\times 2} \stackrel{(a, b, z)}{\longrightarrow} \mathfrak{h}_{0} \oplus \mathfrak{h}_{0} \oplus \mathfrak{h}_{1} \mid \partial_{t} b-\partial_{s} a=l_{2}(a, b)+\mathrm{d} z,\right. \\
b(0, s)=b(1, s)=z(0, s)=z(1, s)=0\} .
\end{array}
$$

There is an equivalence relation $\sim$ defined on $P_{2} \mathfrak{h}:\left(a^{0}, b^{0}, z^{0}\right) \sim\left(a^{1}, b^{1}, z^{1}\right)$ if and only if there are

$$
(a, b, c):[0,1]^{\times 3} \rightarrow \mathfrak{h}_{0}, \quad(x, y, z):[0,1]^{\times 3} \rightarrow \mathfrak{h}_{1},
$$


such that

$$
\begin{aligned}
\partial_{t} b-\partial_{s} a & =l_{2}(a, b)+\mathrm{d} z, \\
\partial_{t} c-\partial_{u} a & =l_{2}(a, c)+\mathrm{d} y, \\
\partial_{s} c-\partial_{u} b & =l_{2}(b, c)+\mathrm{d} x, \\
\partial_{u} z-\partial_{s} y+\partial_{t} x & =l_{2}(a, x)-l_{2}(b, y)+l_{2}(c, z)
\end{aligned}
$$

with boundary conditions:

$$
c(t, s, u), x(t, s, u),\left.y(t, s, u)\right|_{t=0} \text { or } 1, \text { or } s=0 \text { or } 1=0,\left.\quad z(t, s, u)\right|_{t=0} \text { or } 1=0,
$$

and

$$
\begin{aligned}
& a(t, s, 0)=a^{0}, a(t, s, 1)=a^{1}, b(t, s, 0)=b^{0}, b(t, s, 1)=b^{1}, z(t, s, 0)=z^{0}, z(t, s, 1)=z^{1}, \\
& a(t, 0, u)=a^{0}(t, 0)=a^{1}(t, 0), \quad a(t, 1, u)=a^{0}(t, 1)=a^{1}(t, 1) .
\end{aligned}
$$

Then $P_{2} \mathfrak{h} / \sim \Rightarrow P_{1} \mathfrak{h}$ is a groupoid with the source and target evaluation of $a$ on $s=0$ and $s=1$ respectively. Moreover the 2-group of $\tau_{2}\left(\int \mathfrak{h}\right)$ is exactly the 2-group structure on $P_{2} \mathfrak{h} / \sim \Rightarrow P_{1} \mathfrak{h}$ with vertical multiplication the concatenation with respect to the parameter $s$ and horizontal multiplication the concatenation with respect to the parameter $t$. Later on we will give a reparametrized horizontal multiplication (21) (22) (23) for convenience. However, we notice that reparametrization will not change the class in $P_{2} \mathfrak{h}$ :

LEMMA 2.9. Given an element $(a, b, z) \in P_{2} \mathfrak{h}$ and reparametrizations $\tau_{i}:[0,1] \rightarrow$ $[0,1],(a, b, z) \sim\left(a^{\tau}, b^{\tau}, z^{\tau}\right) \in P_{2} \mathfrak{h}$, where

$$
\begin{aligned}
& a^{\tau}(t, s)=\tau_{1}^{\prime}(t) a\left(\tau_{1}(t), \tau_{2}(s)\right), \quad b^{\tau}(t, s)=\tau_{2}^{\prime}(s) b\left(\tau_{1}(t), \tau_{2}(s)\right), \\
& z^{\tau}(t, s)=\tau_{1}^{\prime}(t) \tau_{2}^{\prime}(s) z\left(\tau_{1}(t), \tau_{2}(s)\right) .
\end{aligned}
$$

Proof. In general, elements in $P_{n} \mathfrak{h}$ are d.g.c.a. morphisms $\wedge^{\bullet} \mathfrak{h} \rightarrow \Omega^{\bullet}\left([0,1]^{\times n}\right)$ with certain boundary conditions, and the homotopies $\sim$ are d.g.c.a. morphisms $\wedge^{\bullet} \mathfrak{h} \rightarrow \Omega^{\bullet}\left([0,1]^{\times(n+1)}\right)$ with certain boundary conditions. We define $h$ : $[0,1]^{\times(n+1)} \rightarrow[0,1]^{\times n}$

$$
h\left(t_{1}, \ldots, t_{n+1}\right):=\left(\left(\left(1-t_{n+1}\right) t_{1}+t_{n+1} \tau_{1}\left(t_{1}\right)\right), \ldots,\left(1-t_{n+1}\right) t_{n}+t_{n+1} \tau_{n}\left(t_{n}\right)\right),
$$

pulling back forms by $h$ provides the desired homotopy. See also [8, Remark 3.10] and [9, Lemma 1.5] for similar treatment.

We first construct our equivalence with the help of a couple of lemmas.

LEMMA 2.10. Let $(a, b, z) \in P_{2} \mathfrak{h}$. Let $\Delta b:[0,1]^{\times 2} \longrightarrow \mathfrak{h}_{1}$ satisfy the following ordinary differential equation

$$
\partial_{t} \Delta b=l_{2}(a, \Delta b)-z
$$


with initial value $\Delta b(0, s)=0$. Denote $b+\mathrm{d} \Delta b$ by $\widetilde{b}$, then we have

$$
\partial_{t} \widetilde{b}-\partial_{s} a=[a, \widetilde{b}]_{\mathfrak{h}_{0}} .
$$

Proof. The conclusion follows from:

$$
\begin{aligned}
\partial_{t} \widetilde{b}-\partial_{s} a-[a, \widetilde{b}]_{\mathfrak{h}_{0}} & =\partial_{t} b+\partial_{t} \mathrm{~d} \Delta b-\partial_{s} a-[a, b]_{\mathfrak{h}_{0}}-[a, \mathrm{~d} \Delta b]_{\mathfrak{h}_{0}} \\
& =\partial_{t} \mathrm{~d} \Delta b+\mathrm{d} z-[a, \mathrm{~d} \Delta b]_{\mathfrak{h}_{0}} \\
& =\mathrm{d}\left(\partial_{t} \Delta b+z-l_{2}(a, \Delta b)\right)=0 .
\end{aligned}
$$

Since $\Delta b(0, s)=0$, we have $\widetilde{b}(0, s)=b(0, s)+\mathrm{d} \Delta b(0, s)=0$. But $\widetilde{b}(1, s)=b(1, s)+$ $\mathrm{d} \Delta b(1, s)=\mathrm{d} \Delta b(1, s)$ is not necessarily zero and this is exactly the obstruction of $a(-, 0)$ and $a(-, 1)$ being homotopic.

PROPOSITION 2.11. With the above notations, the concatenation of $\mathrm{d} \Delta b(1,-)$ and $a(-, 0)$ is homotopic to a $(-, 1)$ in $P \mathfrak{h}_{0}$, i.e. we have

$$
\mathrm{d} \Delta b(1,-) \odot a(-, 0) \sim a(-, 1) .
$$

where the concatenation $\odot$ of two paths, $a(t)$ and $b(t)$ is defined as follows:

$$
a(t) \odot b(t)=\left\{\begin{array}{ll}
2 \tau^{\prime}(t) b(\tau(2 t)) & 0 \leq t \leq \frac{1}{2} \\
2 \tau^{\prime}(t) a(\tau(2 t)) & \frac{1}{2} \leq t \leq 1
\end{array},\right.
$$

with a cut-off function $\tau:[0,1] \rightarrow[0,1]$ such that

$$
\tau(0)=0, \quad \tau(1)=1, \quad \tau^{\prime}(t)>0, \quad \forall t \in[0,1] .
$$

Proof. Since we have $\partial_{t} \widetilde{b}-\partial_{s} a=[a, \widetilde{b}]$, there exists a family of paths, $g(t, s)$ in the Lie group $H_{0}$ such that

$$
\partial_{t} g(t, s) \cdot g(t, s)^{-1}=a(t, s), \quad \partial_{s} g(t, s) \cdot g(t, s)^{-1}=\widetilde{b}(t, s) .
$$

Since $\widetilde{b}(0, s)=0$, we know that $g(0, s)$ is fixed. Since $\widetilde{b}(1, s) \neq 0, g(1, s)$ is not a constant path in $H_{0}$. So $g(t, 0)$ and $g(t, 1)$ are not homotopic. However, it is obvious that the concatenation of $g(1, s)$ and $g(t, 0)$ is homotopic to $g(t, 1)$ in the Lie group $H_{0}$. Therefore, the corresponding $\mathfrak{h}_{0}$-paths are $\mathfrak{h}_{0}$-homotopic.

LEMMA 2.12. Let $\mathfrak{h}_{0}$ and $\mathfrak{h}_{1}$ be two Lie algebras, $\phi: \mathfrak{h}_{0} \longrightarrow \operatorname{Der}\left(\mathfrak{h}_{1}\right)$ a Lie algebra morphism, i.e. Lie algebra $\mathfrak{h}_{0}$ acts on Lie algebra $\mathfrak{h}_{1}$ as a derivation. Let $\varphi: H_{0} \longrightarrow$ Aut $\left(\mathfrak{h}_{1}\right)$ be the Lie group morphism which integrates $\phi$, and $h \in H_{0}$ represented by $a(t) \in P \mathfrak{h}_{0}$, i.e. $h=[a(t)]$. Then for any $v \in \mathfrak{h}_{1}$ and $v(s) \in P \mathfrak{h}_{1}$, we have 
(1) $\varphi_{h}(v)=w(1)$, where $w(t) \in P \mathfrak{h}_{1}$ is the solution of the following ODE:

$$
\frac{\mathrm{d}}{\mathrm{d} t} w(t)=\phi_{a(t)} w(t)
$$

with the initial value $w(0)=v$.

(2) $\varphi_{h} v(s)=w(1, s)$, where $w(t, s)$ is the solution of the following $O D E$ :

$$
\partial_{t} w(t, s)=\phi_{a(t)} w(t, s)
$$

with the initial value $w(0, s)=v(s)$.

Consequently, the corresponding group action of $H_{0}$ on $H_{1}$, say $\Phi: H_{0} \longrightarrow \operatorname{Aut}\left(H_{1}\right)$ is given by:

$$
\Phi_{h}([v(s)])=\left[\varphi_{h} v(s)\right]=[w(1, s)] .
$$

Proof. Let $h(t):[0,1] \longrightarrow H_{0}$ be a path such that $h(0)=e, h(1)=h$ and $a(t)=$ $\dot{h}(t) h(t)^{-1}$. Then, we have $\phi_{a(t)}=\dot{\varphi}_{h(t)} \varphi_{h(t)}^{-1}$, which implies that $\phi_{a(t)} \circ \varphi_{h(t)}(v)=$ $\dot{\varphi}_{h(t)}(v)$. Take $w(t)=\varphi_{h(t)}(v)$, then $w(t)$ satisfies the following ODE:

$$
\dot{w}(t)=\phi_{a(t)} w(t)
$$

with the initial value $w(0)=\varphi_{h(0)}(v)=\varphi_{e}(v)=v$. Obviously, $\varphi_{h}(v)=\varphi_{h(1)}(v)=w(1)$. This completes the proof of item (1). Item (2) can be proved similarly.

For bigons $\bullet \frac{a^{2}}{a_{1}}$ and $\bullet$, which represent $(a, b, z)$, $\left(a^{\dagger}, b^{\dagger}, z^{\dagger}\right) \in P_{2} \mathfrak{h}$, respectively, assume that $\Delta b$ and $\Delta b^{\dagger}$ are the corresponding solutions of (15) respectively. We reparametrized the concatenation with respect to $t$, namely the bigon of horizontal multiplication

$$
\begin{aligned}
& a^{\ddagger}(t, s)=\left\{\begin{array}{cc}
a^{\dagger}(t, 2 s), & 0 \leq t \leq 1, \quad 0 \leq s \leq \frac{1}{2} \\
a_{1}^{\dagger}(t), & 0 \leq t \leq 1, \quad \frac{1}{2} \leq s \leq 1 \\
a_{0}(t-1), & 1 \leq t \leq 2, \quad 0 \leq s \leq \frac{1}{2} \\
a(t-1,2 s-1), & 1 \leq t \leq 2, \quad \frac{1}{2} \leq s \leq 1
\end{array}\right. \\
& b^{\ddagger}(t, s)=\left\{\begin{array}{cc}
2 b^{\dagger}(t, 2 s), & 0 \leq t \leq 1, \quad 0 \leq s \leq \frac{1}{2} \\
0, & 0 \leq t \leq 1, \quad \frac{1}{2} \leq s \leq 1 \\
0, & 1 \leq t \leq 2, \quad 0 \leq s \leq \frac{1}{2} \\
2 b(t-1,2 s-1), & 1 \leq t \leq 2, \quad \frac{1}{2} \leq s \leq 1,
\end{array}\right.
\end{aligned}
$$




$$
z^{\ddagger}(t, s)=\left\{\begin{array}{ccc}
2 z^{\dagger}(t, 2 s), & 0 \leq t \leq 1, & 0 \leq s \leq \frac{1}{2} \\
0, & 0 \leq t \leq 1, & \frac{1}{2} \leq s \leq 1 \\
0, & 1 \leq t \leq 2, & 0 \leq s \leq \frac{1}{2} \\
2 z(t-1,2 s-1), & 1 \leq t \leq 2, & \frac{1}{2} \leq s \leq 1,
\end{array}\right.
$$

and denote by $\Delta b^{\ddagger}$ the corresponding solution of (15).

LEMMA 2.13. With the above notations, we have

$$
\Delta b^{\ddagger}(2, s)=\Delta b(1, s) \odot w(1, s),
$$

where $w(t, s):[0,1]^{\times 2} \longrightarrow \mathfrak{h}_{1}$ is the solution of the following ODE:

$$
\partial_{t} w(t, s)=l_{2}\left(a_{0}(t), w(t, s)\right)
$$

with the initial value $w(0, s)=\Delta b^{\dagger}(1, s)$.

Proof. We prove a more general formula:

$$
\Delta b^{\ddagger}(t, s)=\left\{\begin{array}{cc}
0 \odot_{s} \Delta b^{\dagger}(t, s), & 0 \leq t \leq 1 \\
\Delta b(t-1, s) \odot_{s} w(t-1, s) & 1 \leq t \leq 2 .
\end{array}\right.
$$

When $0 \leq t \leq 1$,

$$
\Delta b^{\ddagger}(t, s)=\left\{\begin{array}{cc}
2 \Delta b^{\dagger}(t, s), & 0 \leq s \leq \frac{1}{2} \\
0 & \frac{1}{2} \leq s \leq 1 .
\end{array}\right.
$$

Then, when $0 \leq s \leq \frac{1}{2}, \Delta b^{\ddagger}(t, s)$ satisfies (15) since $\Delta b^{\dagger}(t, s)$ does; when $\frac{1}{2} \leq s \leq 1$, $\Delta b^{\ddagger}(t, s)=0$ obviously satisfies (15).

When $1 \leq t \leq 2$, by straightforward computations, we have

$$
\begin{aligned}
\partial_{t} & \Delta b^{\ddagger}(t, s) \\
& =\partial_{t} \Delta b(t-1, s) \odot_{s} \partial_{t} w(t-1, s) \\
& \left.=\left(l_{2}(a(t-1, s), \Delta b(t-1, s))-z(t-1, s)\right)\right) \odot_{s} l_{2}\left(a_{0}(t-1), w(t-1, s)\right) \\
& = \begin{cases}2 l_{2}\left(a_{0}(t-1), w(t-1,2 s)\right), & 0 \leq s \leq \frac{1}{2}, \\
2 l_{2}(a(t-1,2 s-1), \Delta b(t-1,2 s-1))-2 z(t-1,2 s-1), & \frac{1}{2} \leq s \leq 1 . \\
& =l_{2}\left(a^{\ddagger}(t, s), \Delta b^{\ddagger}(t, s)\right)-z^{\ddagger}(t, s) .\end{cases}
\end{aligned}
$$

The last equality holds because when $1 \leq t \leq 2,0 \leq s \leq \frac{1}{2}, b^{\ddagger}(t, s)=0$.

Define $\Psi_{1}: P_{2} \mathfrak{h} \longrightarrow H_{0} \ltimes H_{1}$ by

$$
\Psi_{1}\left(\bullet \frac{a_{0}}{z \sqrt{a_{1}}} \bullet\right)=\left(\left[a_{0}\right],[\Delta b(1, s)]\right),
$$


in which $\Delta b$ is the unique solution of (15) with the initial value $\Delta b(0, s)=0$. To see that $\Psi_{1}$ is well defined, for two elements $\left(a^{0}, b^{0}, z^{0}\right)$ and $\left(a^{1}, b^{1}, z^{1}\right)$ in $P_{2} \mathfrak{h}$ equivalent through $(a, b, c, x, y, z)$,

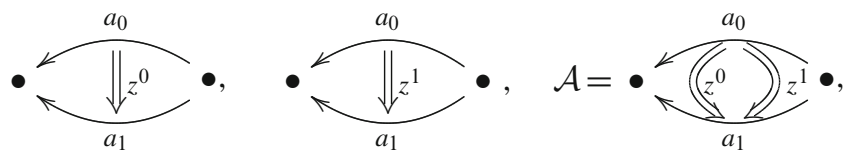

we need to prove that $\Delta b(1, s, 0)$ and $\Delta b(1, s, 1)$ are homotopic in the Lie algebra $\mathfrak{h}_{1}$. This follows from the next lemma.

LEMMA 2.14. Let $(a, b, c, x, y, z)$ be as above. Let $\Delta b, \Delta c:[0,1]^{\times 3} \rightarrow \mathfrak{h}_{1}$ be the solution of the following ordinary differential equations:

$$
\begin{aligned}
& \partial_{t} \Delta b=l_{2}(a, \Delta b)-z, \\
& \partial_{t} \Delta c=l_{2}(a, \Delta c)-y,
\end{aligned}
$$

with the initial value $\Delta b(0, s, u)=\Delta c(0, s, u)=0$, we have

$$
\partial_{s} \Delta c(1, s, u)-\partial_{u} \Delta b(1, s, u)=[\Delta b(1, s, u), \Delta c(1, s, u)]_{\mathfrak{h}_{1}}
$$

and

$$
\Delta c(1,1, u)=0 .
$$

Hence $\Delta b(1, s, 0)$ and $\Delta b(1, s, 1)$ are homotopic.

Proof. Denote $\widetilde{b}=b+\mathrm{d} \Delta b, \widetilde{c}=c+\mathrm{d} \Delta c$, by Lemma 2.10, we have

$$
\begin{aligned}
\partial_{t} \widetilde{b}-\partial_{s} a & =[a, \widetilde{b}]_{\mathfrak{h}_{0}}, \\
\partial_{t} \widetilde{c}-\partial_{u} a & =[a, \widetilde{c}]_{\mathfrak{h}_{0}} .
\end{aligned}
$$

Denote $\bar{a}=a, \bar{b}=b+\Delta b$ and $\bar{c}=c+\Delta c$. By (27) and (44), we have ${ }^{1}$

$$
\begin{aligned}
\partial_{t} \bar{b}-\partial_{s} \bar{a} & =[a, b]_{\mathfrak{h}_{0}}+\mathrm{d} z+l_{2}(a, \Delta b)-z \\
& =[\bar{a}, \bar{b}]_{\mathfrak{h}_{0} \oplus \mathfrak{h}_{1}}+\mathrm{d} z-z .
\end{aligned}
$$

Similarly, we have

$$
\partial_{t} \bar{c}-\partial_{u} \bar{a}=[\bar{a}, \bar{c}]_{\mathfrak{h}_{0} \oplus \mathfrak{h}_{1}}+\mathrm{d} y-y .
$$

Next we prove that

$$
\partial_{s} \bar{c}-\partial_{u} \bar{b}=[\bar{b}, \bar{c}]_{\mathfrak{h}_{0} \oplus \mathfrak{h}_{1}}+\mathrm{d} x-x .
$$

\footnotetext{
${ }^{1}$ See (2) for the definition $[\cdot, \cdot] \mathfrak{h}_{0} \oplus \mathfrak{h}_{1}$.
} 
By straightforward computations, we have

$$
\begin{aligned}
& \partial_{t}\left(\partial_{s} \bar{c}-\partial_{u} \bar{b}-[\bar{b}, \bar{c}]_{\left.\mathfrak{h}_{0} \oplus \mathfrak{h}_{1}\right)}\right.
\end{aligned}
$$

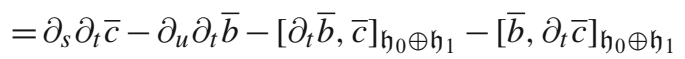

$$
\begin{aligned}
& =\partial_{s}\left(\partial_{u} \bar{a}+[\bar{a}, \bar{c}]_{\mathfrak{h}_{0} \oplus \mathfrak{h}_{1}}+\mathrm{d} y-y\right)-\left[\partial_{t} \bar{b}, \bar{c}\right]_{\mathfrak{h}_{0} \oplus \mathfrak{h}_{1}} \\
& -\partial_{u}\left(\partial_{s} \bar{a}+[\bar{a}, \bar{b}]_{\mathfrak{h}_{0} \oplus \mathfrak{h}_{1}}+\mathrm{d} z-z\right)-\left[\bar{b}, \partial_{t} \bar{c}\right]_{\mathfrak{h}_{0} \oplus \mathfrak{h}_{1}} \\
& =\left[\partial_{s} \bar{a}, \bar{c}\right]_{\mathfrak{h}_{0} \oplus \mathfrak{h}_{1}}-\left[\partial_{t} \bar{b}, \bar{c}\right]_{\mathfrak{h}_{0} \oplus \mathfrak{h}_{1}}+\left[\bar{a}, \partial_{s} \bar{c}\right]_{\mathfrak{h}_{0} \oplus \mathfrak{h}_{1}}+\partial_{s}(\mathrm{~d} y-y) \\
& -\left[\partial_{u} \bar{a}, \bar{b}\right]_{\mathfrak{h}_{0} \oplus \mathfrak{h}_{1}}-\left[\bar{b}, \partial_{t} \bar{c}\right]_{\mathfrak{h}_{0} \oplus \mathfrak{h}_{1}}-\left[\bar{a}, \partial_{u} \bar{b}\right]_{\mathfrak{h}_{0} \oplus \mathfrak{h}_{1}-\partial_{u}(\mathrm{~d} z-z)} \\
& =[\bar{c}, \mathrm{~d} z-z]_{\mathfrak{h}_{0} \oplus \mathfrak{h}_{1}}-[\bar{b}, \mathrm{~d} y-y]_{\mathfrak{h}_{0} \oplus \mathfrak{h}_{1}}+\left[\bar{a}, \partial_{s} \bar{c}-\partial_{u} \bar{b}-[\bar{b}, \bar{c}]_{\mathfrak{h}_{0} \oplus \mathfrak{h}_{1}}\right]_{\mathfrak{h}_{0} \oplus \mathfrak{h}_{1}} \\
& +\partial_{s}(\mathrm{~d} y-y)-\partial_{u}(\mathrm{~d} z-z) \text {. }
\end{aligned}
$$

Meanwhile, we have

$$
\begin{aligned}
{[\bar{c}, \mathrm{~d} z-z]_{\mathfrak{h}_{0} \oplus \mathfrak{h}_{1}} } & =[c+\Delta c, \mathrm{~d} z-z]_{\mathfrak{h}_{0} \oplus \mathfrak{h}_{1}} \\
& =\mathrm{d} l_{2}(c, z)-l_{2}(c, z), \\
{[\bar{b}, \mathrm{~d} y-y]_{\mathfrak{h}_{0} \oplus \mathfrak{h}_{1}} } & =[b+\Delta b, \mathrm{~d} y-y]_{\mathfrak{h}_{0} \oplus \mathfrak{h}_{1}} \\
& =\mathrm{d} l_{2}(b, y)-l_{2}(b, y),
\end{aligned}
$$

By (37), (38), (36) and (14), we obtain that

$$
\partial_{t}\left(\partial_{s} \bar{c}-\partial_{u} \bar{b}-[\bar{b}, \bar{c}]_{\mathfrak{h}_{0} \oplus \mathfrak{h}_{1}}-\mathrm{d} x+x\right)=\left[\bar{a}, \partial_{s} \bar{c}-\partial_{u} \bar{b}-[\bar{b}, \bar{c}]_{\mathfrak{h}_{0} \oplus \mathfrak{h}_{1}}-\mathrm{d} x+x\right]_{\mathfrak{h}_{0} \oplus \mathfrak{h}_{1}} .
$$

Since we have

$$
\left.\left(\partial_{s} \bar{c}-\partial_{u} \bar{b}-[\bar{b}, \bar{c}]_{\mathfrak{h}_{0} \oplus \mathfrak{h}_{1}}-\mathrm{d} x+x\right)\right|_{t=0}=0,
$$

by the uniqueness of solutions of ordinary differential equations, (39) implies (34).

Since $b(1, s, u)=c(1, s, u)=0$, we have

$$
\partial_{s} \Delta c(1, s, u)-\partial_{u} \Delta b(1, s, u)=[\Delta b(1, s, u), \Delta c(1, s, u)]_{\mathfrak{h}_{1}} .
$$

By the initial value condition $a(t, 1, u)=a(t, 1,0)$, for any $u$, since $\bar{a}=a$, we have $\partial_{u} \bar{a}(t, 1, u)=0$. By (33) and $c(t, 1, u)=0$, we have

$$
\partial_{t} \bar{c}(t, 1, u)=[\bar{a}(t, 1, u), \bar{c}(t, 1, u)]_{\mathfrak{h}_{0} \oplus \mathfrak{h}_{1}} .
$$

Since $\bar{c}(0,1, u)=0$, it follows that $\bar{c}(t, 1, u)=0$, which implies that $\Delta c(t, 1, u)=0$ and thus $\Delta c(1,1, u)=0$. Therefore, $\Delta b(1, s, 0)$ and $\Delta b(1, s, 1)$ are homotopic.

Morita equivalence are defined for $n$-groupoids in an arbitrary category with a certain Grothendieck pretopology in [30]. We adapt this notation to our situation: a morphism $F: C \rightarrow C^{\prime}$ of Lie 2-group is a hypercover and denoted by $F: C \stackrel{\sim}{\rightarrow} C^{\prime}$, if $F_{0}: C_{0} \rightarrow C_{0}^{\prime}$ is a surjective submersion, and the natural map

$$
C_{1} \rightarrow C_{1}^{\prime} \times C_{0}^{\prime} \times C_{0}^{\prime} C_{0} \times C_{0}
$$


is an isomorphism. Moreover, $C$ and $C^{\prime}$ are Morita equivalent, if there is another Lie 2-group $C^{\prime \prime}$ such that there are hypercovers $C \stackrel{\sim}{\leftarrow} C^{\prime \prime} \stackrel{\sim}{\rightarrow} C^{\prime}$. A generalized morphism between Lie 2-groups is a span of morphisms $C \stackrel{\sim}{\leftarrow} C^{\prime \prime} \rightarrow C^{\prime}$.

With the above preparations, we have

THEOREM 2.15. There is a Lie 2-group Morita equivalence given by a morphism $\left(\Psi_{0}, \Psi_{1}, \Psi_{2}=i d\right)$ :

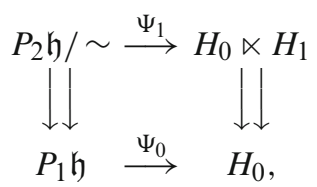

where $\Psi_{0}(a(t))=[a(t)]$, which is the equivalence class of the path a(t) and $\Psi_{1}$ is given by (26).

Remark 2.16. tHERE is an integration obstruction proved in [14], that is, the quotient $P_{2} \mathfrak{h} / \sim$ might not be representable as a Banach manifold unless a certain obstruction class vanishes. In this theorem, we show directly (Proposition 2.18) that $\tau_{2}\left(\int \mathfrak{h}\right)$ is always representable.

We prove it by several steps.

LEMMA 2.17. The above morphism $\left(\Psi_{0}, \Psi_{1}, \Psi_{2}\right)$ is a 2-group morphism.

Proof. Obviously, $\left(\Psi_{1}, \Psi_{0}\right)$ respects the source and target maps. It is not hard to see that $\left(\Psi_{1}, \Psi_{0}\right)$ is a morphism with respect to the vertical multiplication. In fact, for $\bullet \frac{a^{\prime}}{a_{1}} \bullet P^{2} \mathfrak{h}$, assume that $\Delta b, \Delta b^{\sharp}$ are the corresponding solutions of (15), respectively. By definition, we have

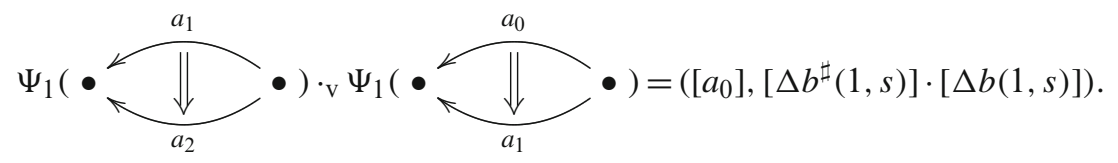

On the other hand, it is straightforward to see that $\Delta b^{\sharp} \odot \Delta b$ is the solution of (15) for the bigon $\bullet \frac{a_{1} \Downarrow}{\sqrt{a_{2}}} \bullet$. Therefore, we have $\Psi_{1}\left(\frac{a_{0}}{a_{a_{2}} \Downarrow} \bullet\right)=$ 
$\left(\left[a_{0}\right],\left[\Delta b^{\sharp}(1, s) \odot \Delta b(1, s)\right]\right)$, which implies that

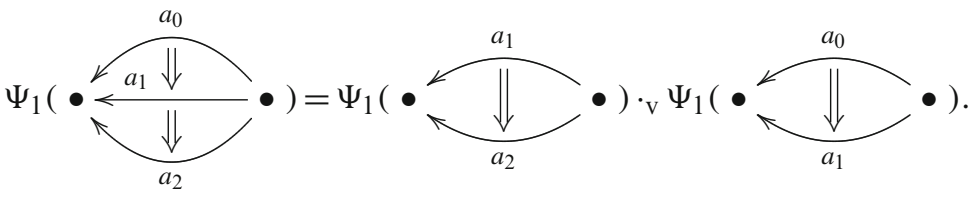

Next we prove that $\left(\Psi_{1}, \Psi_{0}\right)$ is also a morphism with respect to the horizontal multiplication. By (6), we have

$$
\begin{aligned}
& \Psi_{1}\left({ }_{a_{1}}^{\bullet}\right)_{{ }_{h}}^{a_{0}} \\
& =\left(\left[a_{0}\right],[\Delta b(1, s)]\right) \cdot \mathrm{h}\left(\left[a_{0}^{\dagger}\right],\left[\Delta b^{\dagger}(1, s)\right]\right) \\
& \left.\left.\bullet a_{0}^{\dagger}\right],[\Delta b(1, s)] \cdot \Phi_{\left[a_{0}\right]}\left(\left[\Delta b^{\dagger}(1, s)\right]\right)\right),
\end{aligned}
$$

where $\Phi$ is given by (20), which integrates the action of $\mathfrak{h}_{0}$ on $\mathfrak{h}_{1}$.

On the other hand, by Lemma 2.13 and Lemma 2.12, we have

$$
\begin{aligned}
& =\left(\left[a_{0} \odot a_{0}^{\dagger}\right],\left[\Delta b^{\dagger}(2, s)\right]\right) \\
& =\left(\left[a_{0} \odot a_{0}^{\dagger}\right],[\Delta b(1, s) \odot w(1, s)]\right) \\
& =\left(\left[a_{0} \odot a_{0}^{\dagger}\right],[\Delta b(1, s)] \cdot \Phi_{\left[a_{0}\right]}\left(\left[\Delta b^{\dagger}(1, s)\right]\right)\right),
\end{aligned}
$$

which implies that

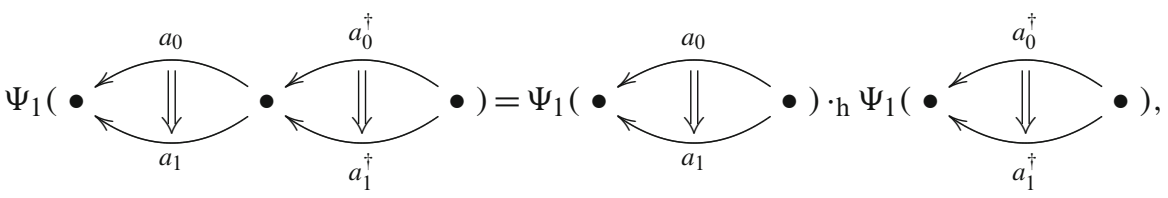

i.e., $\Psi_{1}$ is a morphism with respect to the horizontal multiplication.

Finally, since the right-hand side of (40) is a strict 2-group and $\left(\Psi_{1}, \Psi_{0}\right)$ preserves the horizontal multiplication strictly, condition (7) reduces to

$$
\Psi_{1}\left(a_{a_{3}, a_{2}, a_{1}}\right)=\left(\left[\left(a_{3} \odot a_{2}\right) \odot a_{1}\right], 1_{H_{1}}\right) .
$$

This holds obviously because $a_{a_{3}, a_{2}, a_{1}}$ being a reparametrization between $\left(a_{3} \odot\right.$ $\left.a_{2}\right) \odot a_{1}$ and $a_{3} \odot\left(a_{2} \odot a_{1}\right)$ must be a homotopy by [9, Lemma 1.5]. Similarly, condition (8) holds.

It is clear that $\Psi_{0}$ sends any smooth path in $P \mathfrak{h}$ to a smooth path in $H_{0}$. Moreover, for a smooth family of homotopies (parametrized by $u$ ) $A^{u}=a^{u}(t, s) \mathrm{d} t+$ 
$b^{u}(t, s) \mathrm{d} s$, the solution $\Delta b^{u}(t, s)$ of (15) depends smoothly on $u$. Thus, both $\Phi_{0}$ and $\Phi_{1}$ are smooth.

PROPOSITION 2.18. The natural map

$$
\begin{aligned}
\varpi: P_{2} \mathfrak{h} / & \rightarrow H_{0} \times H_{1} \times H_{0} \times H_{0} \\
{[(a, b, z)] } & \mapsto([a(-, 0)],[\Delta b], a(-, 0), a(-, 1))
\end{aligned}
$$

is an isomorphism.

We first remark that $P_{1} \mathfrak{h}=P \mathfrak{h}_{0}$ and $H_{0}$ is a quotient of $P \mathfrak{h}$, thus $\Psi_{0}: P_{1} \mathfrak{h} \rightarrow H_{0}$ is a surjective submersion of Banach manifolds. Thus this proposition will automatically imply that $P_{2} \mathfrak{h} / \sim$ is representable and hence $\tau_{2}\left(\int \mathfrak{h}\right)$ is a Lie 2-group. The morphism we demonstrate in last lemma will further be a Lie 2-group morphism.

Now we prove this lemma by constructing an inverse morphism. We notice that the Lie group $H_{1}=P^{0} \mathfrak{h}_{1} / \sim$. Given an element $\left(h_{0}, h_{1}, a_{0}, a_{1}\right)$ on the left hand side, we take a representative $\Delta b(s) \in P^{0} \mathfrak{h}_{1}$ of $h_{1}$, then there are $a(t, s), \widetilde{b}(t, s)$ satisfying $\mathfrak{h}_{0}$-homotopy equation (16) and the boundary conditions as in Lemma 2.10. We extend $\Delta b(s)$ to a morphism $\Delta b(t, s):[0,1]^{\times 2} \rightarrow \mathfrak{h}_{1}$ such that

$$
\begin{gathered}
\Delta b(1, s)=\Delta b(s), \quad \Delta b(0, s)=0,\left.\quad \partial_{t}\right|_{t=0} \Delta b(t, s)=0, \\
\left.\partial_{t}\right|_{t=1} \Delta b(t, s)=l_{2}(a(1, s), \Delta(s)) .
\end{gathered}
$$

Such extension always exists. For example, we take

$$
\Delta b(t, s)=\alpha(t) l_{2}(a(1, s), \Delta b(s))+\beta(t) \Delta b(s),
$$

with $\alpha(0)=\alpha(1)=\beta(0)=\alpha^{\prime}(0)=\beta^{\prime}(0)=\beta^{\prime}(1)=0$, and $\alpha^{\prime}(1)=\beta(1)=1$. We take

$$
z(t, s):=l_{2}(a(t, s), \Delta b(t, s))-\partial_{t} \Delta b(t, s), \quad b:=\widetilde{b}-\mathrm{d} \Delta b .
$$

Then

$$
\partial_{t} b-\partial_{s} a-l_{2}(a, b)-\mathrm{d} z=0, \quad z(0, s)=z(1, s)=0, \quad b(1, s)=b(0, s)=\Delta b(0, s)=0,
$$

by construction. Thus we may define a map

$$
\zeta: H_{0} \times H_{1} \times_{H_{0} \times H_{0}} P_{1} \mathfrak{h} \times P_{1} \mathfrak{h} \rightarrow P_{2} \mathfrak{h} / \sim, \quad\left(\left[a_{0}\right],[\Delta b], a_{0}, a_{1}\right) \mapsto[(a, b, z)] .
$$

LEMMA 2.19. The map $\zeta$ is well defined.

Proof. If we take another representative $\Delta b^{1} \in P^{0} \mathfrak{h}_{1}$ which is equivalent to $\Delta b$ in $P^{0} \mathfrak{h}_{1}$ via $\Delta b(s, u)$ and $\Delta c(s, u)$, that is

$$
\partial_{s} \Delta c-\partial_{u} \Delta b=[\Delta b, \Delta c]_{\mathfrak{h}_{1}}, \quad \Delta c(0, u)=\Delta c(1, u)=\Delta b(0, u)=\Delta b(1, u)=0,
$$


and $a_{1} \sim \mathrm{d}(\Delta b) \odot a_{0}$ via $a(t, s), \widetilde{b}(t, s)$ and $a_{1} \sim \mathrm{d}\left(\Delta b^{1}\right) \odot a_{0}$ via $a^{1}(t, s), \widetilde{b}^{1}(t, s)$. Since $\pi_{2}\left(H_{0}\right)=0$, there is no higher obstruction between $\mathfrak{h}_{0}$-homotopies from being homotopic, so $(a(t, s), \widetilde{b}(t, s))$ and $\left(a^{1}(t, s), \widetilde{b}^{1}(t, s)\right)$ must be homotopic via a certain homotopy

$$
a(t, s, u), \quad \widetilde{b}(t, s, u), \quad \widetilde{c}(t, s, u) \quad \in \mathfrak{h}_{0} .
$$

with boundary conditions

$$
\begin{aligned}
& a(t, s, 0)=a(t, s), \quad a(t, s, 1)=a^{1}(t, s), \\
& \widetilde{b}(t, s, 0)=\widetilde{b}(t, s), \quad \widetilde{b}(t, s, 1)=\widetilde{b}^{1}(t, s), \quad \widetilde{b}(0, s, u)=0, \quad \widetilde{b}(1, s, u)=\mathrm{d}(\Delta b(s, u)), \\
& \widetilde{c}(0, s, u)=0, \quad \widetilde{c}(1, s, u)=\mathrm{d}(\Delta c(s, u)), \quad \widetilde{c}(t, 0, u)=0, \quad \widetilde{c}(t, 1, u)=0 .
\end{aligned}
$$

Now we repeat the construction of $z(t, s)$ for each $u$, and we obtain $z(t, s, u)$ and $b(t, s, u)$ with correct boundary conditions satisfying (11). We need to show that $\left.\left.(a, b, z)\right|_{u=0} \sim(a, b, z)\right|_{u=1}$.

Firstly, by a similar method, we construct $y(t, s, u)$ and $c(t, s, u)$ with correct boundary conditions ${ }^{2}$ and satisfying (12). Then $\partial_{u} \widetilde{b}-\partial_{s} \widetilde{c}=[\widetilde{c}, \widetilde{b}]_{\mathfrak{h}_{0}}$ implies that if we take

$$
x(t, s, u)=l_{2}(\Delta b, c)+l_{2}(b, \Delta c)+\partial_{u} \Delta b-\partial_{s} \Delta c+l_{2}(\mathrm{~d}(\Delta b), \Delta c),
$$

we will have (13). The boundary condition $x(0, s, u)=0$ is obvious. The boundary condition $x(1, s, u)=0$ is implied by (43). Implied by the boundary condition in (43), the extension $\Delta b(t, s, u)$ from $\Delta b(s, u)$ according to (42) for each $u$ satisfies $\left.\Delta b(t, s, u)\right|_{s=0,1}=0$. This implies the boundary condition $\left.x\right|_{s=0,1}=0$.

By straightforward computations, we have

$$
\begin{aligned}
\partial_{u} z- & \partial_{s} y+\partial_{t} x \\
= & \partial_{u}\left(l_{2}(a, \Delta b)-\partial_{t} \Delta b\right)-\partial_{s}\left(l_{2}(a, \Delta c)-\partial_{t} \Delta c\right) \\
& +\partial_{t}\left(l_{2}(\Delta b, c)+l_{2}(b, \Delta c)+\partial_{u} \Delta b-\partial_{s} \Delta c+l_{2}(\mathrm{~d}(\Delta b), \Delta c)\right) \\
= & l_{2}\left(\partial_{u} a, \Delta b\right)+l_{2}\left(a, \partial_{u} \Delta b\right)-\partial_{u} \partial_{t} \Delta b-l_{2}\left(\partial_{s} a, \Delta c\right)-l_{2}\left(a, \partial_{s} \Delta c\right)+\partial_{s} \partial_{t} \Delta c \\
& +l_{2}\left(\partial_{t} \Delta b, c\right)+l_{2}\left(\Delta b, \partial_{t} c\right)+l_{2}\left(\partial_{t} b, \Delta c\right)+l_{2}\left(b, \partial_{t} \Delta c\right)+\partial_{t} \partial_{u} \Delta b-\partial_{t} \partial_{s} \Delta c \\
& +l_{2}\left(\partial_{t} \mathrm{~d}(\Delta b), \Delta c\right)+l_{2}\left(\mathrm{~d}(\Delta b), \partial_{t} \Delta c\right) \\
= & l_{2}\left(\partial_{u} a-\partial_{t} c, \Delta b\right)+l_{2}\left(a, \partial_{u} \Delta b-\partial_{s} \Delta c\right)+l_{2}\left(\partial_{t} b-\partial_{s} a, \Delta c\right) \\
& +l_{2}\left(\partial_{t} \Delta b, c\right)+l_{2}\left(b, \partial_{t} \Delta c\right)+l_{2}\left(\partial_{t} \mathrm{~d}(\Delta b), \Delta c\right)+l_{2}\left(\mathrm{~d}(\Delta b), \partial_{t} \Delta c\right) \\
= & -l_{2}\left(l_{2}(a, c)+\mathrm{d} y, \Delta b\right)+l_{2}\left(a, \partial_{u} \Delta b-\partial_{s} \Delta c\right)+l_{2}\left(l_{2}(a, b)+\mathrm{d} z, \Delta c\right) \\
& +l_{2}\left(\partial_{t} \Delta b, c\right)+l_{2}\left(b, \partial_{t} \Delta c\right)+l_{2}\left(\partial_{t} \mathrm{~d}(\Delta b), \Delta c\right)+l_{2}\left(\mathrm{~d}(\Delta b), \partial_{t} \Delta c\right),
\end{aligned}
$$

${ }^{2}$ This amounts to extend $\Delta c(s, u)$ to $\Delta c(t, s, u)$ such that $\left.\Delta c(t, s, u)\right|_{s=0,1}=0, \Delta c(0, s, u)=$ $0, \Delta c(1, s, u)=\Delta c(s, u)$. The boundary condition is a bit different than the case of $\Delta b$, however with more information $\left.\Delta c\right|_{s=0,1}(s, u)=0$ that $\Delta c$ has than $\Delta b$, the same construction of extension works. 
and

$$
\begin{aligned}
& l_{2}(a, x)-l_{2}(b, y)+l_{2}(c, z) \\
& =l_{2}\left(a, l_{2}(\Delta b, c)+l_{2}(b, \Delta c)+\partial_{u} \Delta b-\partial_{s} \Delta c+l_{2}(\mathrm{~d}(\Delta b), \Delta c)\right) \\
& \quad-l_{2}\left(b, l_{2}(a, \Delta c)-\partial_{t} \Delta c\right)+l_{2}\left(c, l_{2}(a, \Delta b)-\partial_{t} \Delta b\right) .
\end{aligned}
$$

Since $l_{2}$ satisfies the Jacobi identity, the condition (14) is equivalent to

$$
\begin{aligned}
& -l_{2}(\mathrm{~d} y, \Delta b)+l_{2}(\mathrm{~d} z, \Delta c)+l_{2}\left(\partial_{t} \mathrm{~d}(\Delta b), \Delta c\right)+l_{2}\left(\mathrm{~d}(\Delta b), \partial_{t} \Delta c\right) \\
& \quad-l_{2}\left(a, l_{2}(\mathrm{~d} \Delta b, \Delta c)\right)=0 .
\end{aligned}
$$

Compute directly, the left-hand side is equal to

$$
\begin{aligned}
& -l_{2}\left(\mathrm{~d} l_{2}(a, \Delta c), \Delta b\right)+l_{2}\left(\mathrm{~d} \partial_{t} \Delta c, \Delta b\right)+l_{2}\left(\mathrm{~d} l_{2}(a, \Delta b), \Delta c\right)-l_{2}\left(\mathrm{~d} \partial_{t} \Delta b, \Delta c\right) \\
& \quad+l_{2}\left(\partial_{t} \mathrm{~d}(\Delta b), \Delta c\right)+l_{2}\left(\mathrm{~d}(\Delta b), \partial_{t} \Delta c\right)-l_{2}\left(a, l_{2}(\mathrm{~d} \Delta b, \Delta c)\right),
\end{aligned}
$$

which is equal to zero since $l_{2}$ satisfies the Jacobi identity. Thus, (14) holds. Therefore, we have $\left.\left.(a, b, z)\right|_{u=0} \sim(a, b, z)\right|_{u=1}$ through $(a, b, c, x, y, z)$.

It is obvious that $\varpi \circ \zeta=i d$. To finish the proof of Theorem 2.15, we still need to show that $\zeta \circ \varpi=i d$. Given an element $(a, b, z) \in P_{2} \mathfrak{h}$, since $\varpi$ does not depend on the choice of representative, we choose a convenient reparametrization such that $\left.z(t, s)\right|_{s=0,1}=0$. Thus the solution $\Delta b$ in Lemma 2.10 also has $\left.\Delta b(t, s)\right|_{s=0,1}=$ 0 . Following $\varpi$ then $\zeta$, we first restrict $\Delta b$ on $t=1$, then extend it again to all $t$ by (42), thus we might end up with another $\Delta b^{1}$, with the same boundary value, that is when either $t$ or $s$ is 0 or 1 . Thus Theorem 2.15 follows immediately from the following lemma:

LEMMA 2.20. The map $\zeta$ does not depend on the choice of extension with the same boundary value.

Proof. We suppose that there are two such extensions $\Delta b(t, s)$ and $\Delta b^{1}(t, s)$. We connect them by $\Delta b^{u}:=u \Delta b+(1-u) \Delta b^{1}$. Then the corresponding $b:=\widetilde{b}-\mathrm{d} \Delta b$ and $b^{1}:=\widetilde{b}-\mathrm{d} \Delta b^{1}$ are connected by $b^{u}:=\widetilde{b}-\Delta b^{u}$; the corresponding $z$ and $z^{1}$ are connected by $z^{u}:=l_{2}\left(a, \Delta b^{u}\right)-\partial_{t} \Delta b^{u}$. Now we take $a(t, s, u)=a(t, s), b(t, s, u)=$ $b^{u}, c=0, y=0, x=\partial_{u} \Delta b^{u}$, then it is obviously to see that (11), (12) hold. Equation (13) is implied by the fact that $\widetilde{b}$ does not depend on $u$. Equation (14) is implied by the fact that $a$ does not depend on $u$. The boundary condition of $x$ is implied by that of $\Delta b$ and $\Delta b^{1}$. Thus $\left.(a, b, z)\right|_{u=0}$ is homotopic to $\left.(a, b, z)\right|_{u=1}$.

\section{Application on Integration of (Non-Strict) Lie 2-Algebra Morphisms}

Lie's theorem II tells us that Lie algebra morphisms can integrate to Lie group morphisms. As pointed out in [11, Def. 4.2.8] (and also easy to see), an $L_{\infty^{-}}$ morphism between $L_{\infty}$-algebras $f: \mathfrak{g} \rightarrow \mathfrak{h}$ induces a natural map $\int f: \int \mathfrak{g} \rightarrow \int \mathfrak{h}$ 
of Kan complex. Thus applying in the case of Lie 2-algebras, an $L_{\infty}$-morphism between Lie 2-algebras (also called non-strict Lie 2-algebra morphisms) $f: \mathfrak{g} \rightarrow \mathfrak{h}$ can integrate to a 2-group morphism $\tau_{2}\left(\int f\right): \tau_{2}\left(\int g\right) \rightarrow \tau_{2}\left(\int h\right)$. Combining with our result, we have

COROLLARY 3.1. A non-strict Lie 2-algebra morphism $f: \mathfrak{g} \rightarrow \mathfrak{h}$ between two strict Lie 2-algebras integrates to a generalized Lie 2-group morphism

$$
\left(G_{0} \ltimes G_{1} \Rightarrow G_{0}\right) \stackrel{\sim}{\leftarrow} \tau_{2}\left(\int \mathfrak{g}\right) \stackrel{\tau_{2}\left(\int f\right)}{\longrightarrow} \tau_{2}\left(\int \mathfrak{h}\right) \stackrel{\sim}{\rightarrow}\left(H_{0} \ltimes H_{1} \Rightarrow H_{0}\right),
$$

between the corresponding (simply-connected) Lie group crossed modules.

Remark 3.2. It is fairly easy to integrate a strict morphism which consists of Lie algebra morphisms $f_{i}: \mathfrak{g}_{i} \rightarrow \mathfrak{h}_{i}$ preserving all crossed module structures. One only needs to integrate $f_{i}$ individually as a Lie algebra morphism.

The integration of nonstrict morphism is also addressed in the context of butterflies [20]. Butterflies between crossed modules are believed ${ }^{3}$ to be equivalent to generalized morphisms between strict Lie 2-groups.

Finally, we call the generalized morphism above an integration of $f$ based on the fact that $\tau_{2}\left(\int f\right)$ should be considered as a canonical integration. However we do not justify the concept of integration by the inverse procedure, namely differentiation.

Now we concentrate on Lie 2-algebra morphisms from a Lie algebra to a strict Lie 2-algebra. We will see that several interesting objects can be described by such a morphism, including 2-term representations up to homotopy of Lie algebras, non-abelian extensions of Lie algebras and up to homotopy Poisson actions.

We first recall an explicit formulation of $L_{\infty}$-morphism that we will mention in the examples:

DEFINITION 3.3. An $L_{\infty}$-morphism from a Lie algebra $\mathfrak{g}$ to a strict Lie 2-algebra $L_{1} \stackrel{\mathrm{d}}{\longrightarrow} L_{0}$ consists of linear maps $\mu: \mathfrak{g} \longrightarrow L_{0}$ and $\nu: \mathfrak{g} \wedge \mathfrak{g} \longrightarrow L_{1}$ such that the obstruction of $\mu$ being a Lie algebra morphism is given by

$$
\mu[X, Y]_{\mathfrak{g}}-l_{2}(\mu(X), \mu(Y))=\mathrm{d} v(X, Y),
$$

and $v$ satisfies the following condition:

$$
l_{2}(\mu(X), v(Y, Z))+c \cdot p \cdot=v\left([X, Y]_{\mathfrak{g}}, Z\right)+c \cdot p \cdot,
$$

where $c . p$. means cyclic permutations.

\footnotetext{
${ }^{3}$ Private conversation to Noohi.
} 


\section{- 2-term representations up to homotopy of Lie algebras}

Associated to any $k$-term complex of vector spaces $\mathbb{V}$, there is a natural DGLA (differential graded Lie algebra) $\mathfrak{g l}(\mathbb{V})[16,23]$, which plays the same role as $\mathfrak{g l}(V)$ for a vector space $V$ in the classical case. An $L_{\infty}$-module [16] of an $L_{\infty}$-algebra $L$ is given by an $L_{\infty}$-morphism from $L$ to $\mathfrak{g l}(\mathbb{V})$. Associated to any 2-term complex of vector spaces $\mathcal{V}$, by truncation of $\mathfrak{g l}(\mathcal{V})$, we obtain a strict Lie 2-algebra, which we denote by $\operatorname{End}(\mathcal{V})$. The degree 0 part $\operatorname{End}^{0}(\mathcal{V})$ is given by

$$
\operatorname{End}^{0}(\mathcal{V})=\left\{\left(A_{0}, A_{1}\right) \in \operatorname{End}\left(V_{0}, V_{0}\right) \oplus \operatorname{End}\left(V_{1}, V_{1}\right) \mid A_{0} \circ \mathrm{d}=\mathrm{d} \circ A_{1}\right\},
$$

and the degree 1 part $\operatorname{End}^{1}(\mathcal{V})$ is $\operatorname{Hom}\left(V_{0}, V_{1}\right)$. The Lie bracket of $\operatorname{End}(\mathcal{V})$ is given by the commutator and the differential is induced by $\mathrm{d}$. It turns out that for 2-term $L_{\infty}$-modules of a Lie algebra $\mathfrak{g}$, it is enough to look at morphisms to the strict Lie 2-algebra $\operatorname{End}(\mathcal{V})$ :

PROPOSITION 3.4. [16] A 2-term $L_{\infty}$-module of a Lie algebra $\mathfrak{g}$ is given by an $L_{\infty}$-morphism from $\mathfrak{g}$ to $\operatorname{End}(\mathcal{V})$.

A 2-term $L_{\infty}$-module of a Lie algebra $\mathfrak{g}$ is the same as a representation up to homotopy of the Lie algebra $\mathfrak{g}$ on a 2-term complex of vector spaces, see [2,22] for more details. Thus, Corollary 3.1 can be applied to integrate $L_{\infty}$-modules $\mathcal{V}$ of a Lie algebra $\mathfrak{g}$ to that of a Lie group $G$. This is studied further in [23], where the semidirect product $\mathfrak{g} \ltimes \mathcal{V}$ is also integrated. It then has application in integrating omni-Lie algebras and Courant algebroids [24].

\section{- Non-abelian extensions of Lie algebras}

It is well known that abelian extensions of a Lie algebra $\mathfrak{g}$ give rise to a representation of $\mathfrak{g}$ and the equivalence classes of extensions are in one-to-one correspondence with the second cohomology. In the following, we will see that a non-abelian extension of a Lie algebra $\mathfrak{g}$, given by a short exact sequence of Lie algebras

$$
0 \longrightarrow \mathfrak{k} \stackrel{i}{\longrightarrow} \hat{\mathfrak{g}} \stackrel{p}{\longrightarrow} \mathfrak{g} \longrightarrow 0
$$

can be realized as an $L_{\infty}$-morphism from Lie algebra $\mathfrak{g}$ to the strict Lie 2-algebra $\mathfrak{k} \stackrel{\text { ad }}{\longrightarrow} \operatorname{Der}(\mathfrak{k})$ (see Example 2.4).

By choosing a splitting of $p$, we can always assume that $\hat{\mathfrak{g}}=\mathfrak{g} \oplus \mathfrak{k}$ as vector spaces. Then the Lie bracket $[\cdot, \cdot]_{\hat{g}}$ decomposes as below,

$$
\begin{aligned}
{\left[X_{1}+k_{1}, X_{2}+k_{2}\right]_{\hat{\mathfrak{g}}}=} & {\left[X_{1}, X_{2}\right]_{\hat{\mathfrak{g}}}+\left[X_{1}, k_{2}\right]_{\hat{\mathfrak{g}}}-\left[X_{2}, k_{1}\right]_{\hat{\mathfrak{g}}}+\left[k_{1}, k_{2}\right]_{\mathfrak{k}}, } \\
& \forall X_{1}+k_{1}, X_{2}+k_{2} \in \mathfrak{g} \oplus \mathfrak{k} .
\end{aligned}
$$


Since $p$ is a morphism of Lie algebras, there is a linear map $v: \mathfrak{g} \wedge \mathfrak{g} \longrightarrow \mathfrak{k}$ such that

$$
\left[X_{1}, X_{2}\right]_{\hat{\mathfrak{g}}}=\left[X_{1}, X_{2}\right]_{\mathfrak{g}}+v\left(X_{1}, X_{2}\right) .
$$

On the other hand, it is straightforward to see that for any $X \in \mathfrak{g}$, the action $[X, \cdot]_{\hat{\mathfrak{g}}}: \mathfrak{k} \longrightarrow \mathfrak{k}$ is a derivation with respect to the Lie bracket $[\cdot, \cdot]_{\mathfrak{k}}$. Thus $[X, k]_{\hat{\mathfrak{g}}}=$ $\mu(X)(k)$ for some linear map $\mu: \mathfrak{g} \longrightarrow \operatorname{Der}(\mathfrak{k})$. One should be very careful here: $\mu$ is not a Lie algebra morphism!

We rewrite $[\cdot, \cdot]_{\hat{\mathfrak{g}}}$ as

$$
\left[X_{1}+k_{1}, X_{2}+k_{2}\right]_{\hat{\mathfrak{g}}}=\left[X_{1}, X_{2}\right]_{\mathfrak{g}}+\mu\left(X_{1}\right)\left(k_{2}\right)-\mu\left(X_{2}\right)\left(k_{1}\right)+\left[k_{1}, k_{2}\right]_{\mathfrak{k}}+v\left(X_{1}, X_{2}\right) .
$$

The Jacobi identity of $[\cdot, \cdot]_{\hat{\mathfrak{g}}}$ gives,

$$
\begin{aligned}
& \mu\left([X, Y]_{\mathfrak{g}}\right)(k)-[\mu(X), \mu(Y)]_{C}(k)=[v(X, Y), k]_{\mathfrak{k}}, \\
& \mu(X)(v(Y, Z))+c \cdot p \cdot=v\left([X, Y]_{\mathfrak{g}}, Z\right)+c \cdot p . .
\end{aligned}
$$

Moreover, a different splitting $p^{\prime}$ will give a homotopic morphism. We conclude by the following proposition, which we do not claim any originality (see $[1,15,6,26]$ for various similar and more general discussions).

PROPOSITION 3.5. Given two Lie algebras $\mathfrak{g}$ and $\mathfrak{k}$, there is a one-to-one correspondence between the equivalence classes of non-abelian extensions of $\mathfrak{g}$ by $\mathfrak{k}$ and homotopy classes of $L_{\infty}$-morphisms from $\mathfrak{g}$ to the strict Lie 2-algebra $\mathfrak{k} \longrightarrow \operatorname{Der}(\mathfrak{k})$.

Thus apply our result on integration may provide another method to integrate non-abelian extensions of Lie algebras.

\section{- Up to homotopy Poisson actions}

DEFINITION 3.6. [21] An up to homotopy Poisson action of a Lie algebra $\mathfrak{g}$ on a Poisson manifold $(M, \pi)$ is an extension $\mathfrak{g}_{M}$ of $\mathfrak{g}$ by the Lie algebra $C^{\infty}(M)$ (with the Poisson bracket $\{\cdot, \cdot\}_{\pi}$ used as the Lie bracket), such that for every $X \in$ $\mathfrak{g}_{M}$, the map $C^{\infty}(M) \longrightarrow C^{\infty}(M), f \longmapsto[X, f]$ is a derivation (i.e. a vector field).

Let $L_{M}^{\pi}$ denote the DGLA of multi-vector fields $\Gamma(\wedge T M)$ [1], with Schouten bracket $[\cdot, \cdot]_{S}$ and differential $[\pi, \cdot]$.

As stated in [21], another equivalent formulation of up to homotopy Poisson action is an $L_{\infty}$ morphism from $\mathfrak{g}$ to the DGLA $L_{M}^{\pi}$. We further simplify this statement. Denote by $\mathfrak{X}(M)^{\pi}$ the set of vector fields preserving the Poisson structure $\pi$, i.e.,

$$
\mathfrak{X}(M)^{\pi}=\{X \in \mathfrak{X}(M), \quad[X, \pi]=0\} .
$$


By truncation, we obtain a strict Lie 2-algebra $C^{\infty}(M) \stackrel{[\pi, \cdot]}{\longrightarrow} \mathfrak{X}(M)^{\pi}$, of which the degree 1 part is $C^{\infty}(M)$, the degree 0 part is $\mathfrak{X}(M)^{\pi}$ and the differential is [ $\left.\pi, \cdot\right]$. The extension $\mathfrak{g}_{M}$ of $\mathfrak{g}$ by $C^{\infty}(M)$ is totally determined by a linear map $\mu: \mathfrak{g} \longrightarrow$ $\mathfrak{X}(M)^{\pi}$ and a linear map $v: \mathfrak{g} \wedge \mathfrak{g} \longrightarrow C^{\infty}(M)$, which satisfy the following equation:

$$
\begin{aligned}
& \mu\left([X, Y]_{\mathfrak{g}}\right)-[\mu(X), \mu(Y)]_{S}=[\pi, v(X, Y)]_{S}, \\
& \mu(X)(v(Y, Z))+c . p .=v\left([X, Y]_{\mathfrak{g}}, Z\right)+c . p . .
\end{aligned}
$$

Thus, we have

PROPOSITION 3.7. There is a one-to-one correspondence between up to homotopy Poisson actions of Lie algebra $\mathfrak{g}$ on Poisson manifolds $(M, \pi)$ and $L_{\infty}$-morphisms $(\mu, v)$ from $\mathfrak{g}$ to the strict Lie 2-algebra $C^{\infty}(M) \stackrel{[\pi, \cdot]}{\longrightarrow} \mathfrak{X}(M)^{\pi}$.

Remark 3.8. We only need to use the fact $\pi_{2}\left(H_{0}\right)=0$ in the construction of $g$ in the last section. Without this condition, we will still have a morphism even though not a Morita morphism. The space of $\mathfrak{X}(M)^{\pi}$ is infinite dimensional and does not admit a Banach structure. However, there is also certain infinite-dimensional calculus available in this case (see for example [28, App.A]). Thus our result cannot be applied directly, however, certain modification may be applied.

\section{Acknowledgements}

We thank the referee very much for very helpful comments. Both authors give their warmest thanks to Courant Research Centre "Higher Order Structures", Göttingen University, and Jilin University, where this work was done during their visits.

Open Access This article is distributed under the terms of the Creative Commons Attribution Noncommercial License which permits any noncommercial use, distribution, and reproduction in any medium, provided the original author(s) and source are credited.

\section{References}

1. Alekseevsky, D., Michor, P.W., Ruppert, W.: Extensions of Lie algebras, arXiv:math. DG/0005042

2. Abad, C.A., Crainic, M.: Representations up to homotopy of Lie algebroids. J. Reine Angew. Math. 663, 91-126 (2012)

3. Baez, J., Lauda, A.: Higher-dimensional algebra 5: 2-groups. Theory Appl. Categ. 12, 423-491 (2004)

4. Baez, J., Crans, A.S.: Higher-dimensional algebra 6: Lie 2-algebras. Theory Appl. Categ. 12, 492-538 (2004)

5. Baez, J.C., Schreiber, U., et al.: Higher Gauge Theory. In: Davydov, A. (ed.) Categories in Algebra, Geometry and Mathematical Physics. Contemporary Mathematics, vol. 431, pp. 7-30. AMS Providence (2007)

6. Chen, S., Sheng, Y., Zheng, Z.: Non-abelian Extensions of Lie 2-algebras, arXiv:1203.0367. Sci. China. Math. (2012). doi:10.1007/s11425-012-4398-7 
7. Brahic, O.: Extensions of Lie brackets. J. Geom. Phys. 60(2), 352-374 (2010)

8. Brahic, O., Zhu, C.: Lie algebroid fibrations. Adv. Math. 226(4), 3105-3135 (2011)

9. Crainic, M., Fernandes, R.L.: Integrability of Lie brackets. Ann. Math. (2) 157(2), 575-620 (2003)

10. Duistermaat, J., Kolk, J.: Lie Groups, Universitext. Springer, New York (2000)

11. Fiorenza, D., Schreiber, U., Stasheff, J.: Cech cocycles for differential characteristic classes - an infinity-Lie theoretic construction, arXiv:1011.4735v2

12. Forrester-Barker, M.: Group objects and internal categories, math.CT/0212065

13. Getzler, E.: Lie theory for nilpotent $L_{\infty}$-algebras. Ann. Math. (2) 170(1), 271-301 (2009)

14. Henriques, A.: Integrating $L_{\infty}$-algebras. Compos. Math. 144(4), 1017-1045 (2008)

15. Inassaridze, N., Khmaladze, E., Ladra, M.: Non-abelian cohomology and extensions of Lie algebras. J. Lie Theory 18, 413-432 (2008)

16. Lada, T., Mark1, M.: Strongly homotopy Lie algebras. Commun. Algebra 23(6), 21472161 (1995)

17. Li-Bland, D., Ševera, P.: Integration of exact courant algebroids, arXiv:1101.3996

18. Liu, Z.-J., Weinstein, A., Xu, P.: Manin triples for Lie bialgebroids. J. Differ. Geom. 45, 547-574 (1997)

19. Mehta, R.A., Tang, X.: From double Lie groupoids to local Lie 2-groupoids. Bull. Braz. Math. Soc. 42(4), 651-681 (2011)

20. Noohi, B.: Integrating morphisms of Lie 2-algebras, arXiv:0910.1818

21. Severa, P.: Poisson actions up to homotopy and their quantization. Lett. Math. Phys. 77(2), 199-208 (2006)

22. Sheng, Y., Zhu, C.: Semidirect products of representations up to homotopy. Pac. J. Math. 249(1), 211-236 (2011)

23. Sheng, Y., Zhu, C.: Integration of semidirect product Lie 2-algebras. Int. J. Geom. Methods Mod. Phys. 9(5), 1250043 (2012)

24. Sheng, Y., Zhu, C.: Higher extensions of Lie algebroids, arXiv:1103.5920

25. Stasheff, J.: Differential graded Lie algebras, quasi-Hopf algebras and higher homotopy algebras. In: Quantum groups (Leningrad, 1990). Lecture Notes in Mathematics, vol. 1510, pp. 120-137. Springer, Berlin (1992)

26. Stevenson, D.: Schreier theory for Lie 2-algebras. Unpublished work

27. Tseng, H., Zhu, C.: Integrating Lie algebroids via stacks. Compos. Math. 142(1), 251270 (2006)

28. Wockel, C., Zhu, C.: Integrating central extensions of Lie algebras via Lie 2-groups, arXiv: 1204.5583

29. Zhu, C.: Lie II theorem for Lie algebroids via higher groupoids, arXiv:math/0701024

30. Zhu, C.: $n$-Groupoids and Stacky Groupoids. Int. Math. Res. Not. IMRN 21, 40874141 (2009) 